\title{
PROPUESTA METODOLÓGICA PARA EL USO DE LAS TECNOLOGÍAS DE LA INFORMACIÓN Y LAS COMUNICACIONES EN LA ENSEÑANZA DEL PERIODISMO HIPERMEDIA
}

\author{
METHODOLOGICAL PROPOSAL FOR THE USE OF INFORMATION AND \\ COMMUNICATION TECHNOLOGIES (ICTS) IN THE TEACHING OF HYPER- \\ MEDIA JOURNALISM
}

\author{
Karla Beatriz Paredes Galindo ${ }^{1}$ \\ $1^{*}$ Universidad de Camagüey "Ignacio Agramonte Loynaz", Camagüey, Cuba. \\ Email: karla.paredes@reduc.edu.cu (iD https://orcid.org/0000-0001-8006-9689
}

\begin{abstract}
RESUMEN:
Las tecnologías de la información y las comunicaciones (TICS) constituyen una importante herramienta de trabajo para la investigación y la planificación en el campo del periodismo y la comunicación. El nuevo contexto en que Cuba se desenvuelve exige la creación de condiciones para ofrecerle a la sociedad profesionales del periodismo capaces de usar las tecnologías, insertarse activamente en los entornos mediáticos y gestionar proyectos para la transformación social. En el Departamento de Periodismo y Comunicación Social de la Universidad de Camagüey Ignacio Agramonte Loynaz se articulan estrategias en aras de la formación integral de los estudiantes. Desde la Disciplina Comunicación Hipermedia, Tecnología y Sociedad, se desarrollan ejercicios integradores que coadyuvan a la práctica periodística de los futuros profesionales. El objetivo del presente trabajo es argumentar una Propuesta metodológica para la enseñanza del Periodismo Hipermedia, utilizando los blogs como parte de las TICs. Todo esto está en consonancia con el plan de Estudios $\mathrm{E}$ que se implementa en la Carrera desde el año 2017.
\end{abstract}

\section{PALABRAS CLAVE:}

periodismo hipermedia, tecnología, blogs, Universidad de Camagüey
Recibido: $13 / 06 / 2020$

Aceptado: 03/03/2021

\section{ABSRTACT:}

Information and communication technologies (ICTs) are an important working tool for research and planning, in the field of journalism and communication. The new context in which Cuba operates requires creating conditions to offer society professionals in journalism culturally capable of using technologies, actively inserting themselves in media environments, managing projects for social transformation. In the Department of Journalism and Social Communication of the University of Camagüey Ignacio Agramonte Loynaz strategies are articulated for the comprehensive training of students. From the Hypermedia Communication, Technology and Society Discipline, integrative exercises are developed that contribute to the journalistic practice of future professionals. The objective of this work is methodological for the teaching of Hypermedia Journalism, using blogs as part of ICTs. All this is in line with the " $E$ " Study plan that has been implemented in the Degree Program since 2017.

\section{KEYWORDS:}

hypermedia journalism, technology, blogs, University of Camagüey 
익

P U B L I C A N D O

I S S N $133900-93304$

\section{INTRODUCCIÓN}

$\mathrm{L}$

a formación de los profesionales del periodismo se plantea hoy en Cuba en un contexto marcado por la convergencia y diversificación de prácticas, sistemas y escenarios de la producción comunicativa, por procesos de estructuración y transformación incesantes, por la permeabilidad de las fronteras entre lo privado y lo público, lo local y lo global y la presencia de la ciudadanía como productora y consumidora de bienes culturales.

Los medios de comunicación experimentan constantes transmutaciones provocadas también por significativas condicionantes económicas. El periodismo enfrenta dilemas tecnológicos, éticos y profesionales; sin embargo, su función pública continúa siendo altamente valorada a nivel social y el estudio de la especialidad se mantiene entre los diseños curriculares de nivel superior de Cuba y de numerosos países del mundo.

En este sentido, la formación de los estudiantes de periodismo supone la superación de la visión instrumental que dominó durante décadas los espacios académicos, y se expande hacia la generación de soluciones innovadoras integradas en materia de comunicación no solo en los medios, sino también en el sistema empresarial, la administración pública y otros sectores de la economía y la sociedad.

El Departamento de Periodismo y Comunicación Social de la Universidad de Camagüey Ignacio Agramonte Loynaz articula estrategias en aras de la formación integral de los estudiantes. Desde la Disciplina Comunicación Hipermedia, Tecnología y Sociedad, se desarrollan ejercicios integradores que coadyuvan a la práctica periodística de los futuros profesionales. El objetivo del presente trabajo es argumentar una Propuesta metodológica para la enseñanza del Periodismo Hipermedia, utilizando los blogs como parte de las TICs.

El nuevo contexto en que Cuba se desenvuelve exige la creación de condiciones para ofrecerle a la sociedad profesionales culturalmente capaces de usar las tecnologías, insertarse activamente en los entornos mediáticos, gestionar proyectos para la transformación social e intervenir en estrategias de desarrollo hacia sociedades más inclusivas y equitativas.

\section{DESARROLLO}

\section{1- EL PROCESO DE FORMACIÓN INTEGRAL EN LOS ESTUDIANTES UNIVERSITARIOS EN LA UNIVERSIDAD DE CAMAGÜEY IGNACIO AGRAMONTE Y LOYNAZ}

La concepción de la Educación Superior en Cuba, concibe a las universidades como responsables de la formación tanto inicial como permanente. El modelo que se debe defender para la universidad cubana es el de "una universidad moderna, humanista y universalizada, científica, tecnológica e innovadora, integrada a la sociedad, al sector productivo y a las comunidades, y comprometida con el proyecto social de la nación" (Fraga, 2018).

Este modelo de universidad exige el incremento continuo de la calidad y la pertinencia en la formación integral de los estudiantes con énfasis en los valores de la Revolución Cubana, y en la creación, extensión y difusión del conocimiento. La calidad, es el resultado de la conjunción de la excelencia académica y la pertinencia integral. Existe una relación dialéctica entre la excelencia académica y la pertinencia integral, en aras del cumplimiento de la misión de la educación y para garantizar la integralidad en el proceso de formación de los profesionales. La calidad y la pertinencia de la formación en la Educación Superior cubana se sustenta en el trabajo educativo, con énfasis en lo político-ideológico, porque engloba la educación basada en un sistema de valores de la Revolución Cubana que propende a la formación de profesionales que combine una elevada competencia profesional con sólidas convicciones revolucionarias.

La formación integral de los estudiantes universitarios significa preparar a los ciudadanos para la vida; para su desempeño en la sociedad. Un enfoque integral de este proceso permite identificar tres dimensiones esenciales (instructiva, desarrolladora y educativa) (Horruitiner, 2009). Estas dimensiones están indisolublemente unidas entre sí, y se manifiestan a lo largo de toda la carrera. La dimensión desarrolladora que debe poner al estudiante en contacto con el objeto de su profesión y contribuir al desarrollo de los modos de actuación integradoras determinados para cada año académico y por último la dimensión educativa debe prepararlo para vivir en sociedad y ser portador de un sistema de valores.

En el Reglamento de Trabajo Docente Metodológico, más conocido como la Resolución No. 2 del 2018 también se realiza especial énfasis en la formación integral de los estudiantes cuando dictamina "la formación de los profesionales de nivel superior es el proceso que (...) se desarrolla en las instituciones de Educación Superior para garantizar 


\section{삭 \\ $\begin{array}{lllllll}R & E & V & I & S & T & A\end{array}$ \\ P U B L I C A N D O \\ I S S N $133900-93004$}

la preparación integral de los estudiantes universitarios" (MES, 2017) más adelante se explica "que el modelo de formación del profesional de la Educación Superior cubana es de perfil amplio y se sustenta en dos ideas rectoras fundamentales: la unidad entre la educación y la instrucción y el vínculo del estudio con el trabajo" (MES, 2017).

\section{2- PARTICULARIDADES DEL PLAN DE ES- TUDIOS E PARA LA CARRERA DE PERIODIS- MO}

La evolución del campo del periodismo a nivel nacional e internacional y la experiencia acumulada en la formación de profesionales de la prensa, permiten a las carreras de Periodismo en los distintos Centros de Educación Superior (CES) plantearse como objetivo "la formación de egresados aptos para gestionar procesos de comunicación en espacios institucionales y sáciales a diferentes niveles. Estos deberán ser capaces de dominar los lenguajes y las técnicas de los medios de comunicación, producir mensajes periodísticos desde una perspectiva de pensamiento crítico, informado y responsable, y contribuir a la proyección estratégica y el desarrollo sostenible de los propios flujos, proyectos y sistemas de comunicación" (MES, 2017).

El objeto de trabajo de los profesionales del periodismo constituye la información periodística mediática y extramediática, mientras que los modos de actuación del profesional radican en la formación humanística, el dominio de los lenguajes y técnicas de la profesión en los nuevos entornos laborales, el manejo de idiomas extranjeros, el uso eficaz de recursos tecnológicos y una conducta ética. La formación durante los cuatro años habilitan a los egresados para la: producción y socialización de discursos periodísticos e infocomunicativos, análisis y procesamiento de información, investigación periodística e infocomunicativa, planeación estratégica, gestión y evaluación de procesos de infocomunicación y la puesta en práctica de propuestas innovadoras de trabajo.

El campo de acción en el que se mueven los futuros periodistas lo constituyen la producción infocomunicativa en el sistema global, nacional y local de medios, la construcción del discurso periodístico, la gestión estratégica en organizaciones mediáticas y extramediáticas y la investigación científica en comunicación. Entre las esferas de actuación de los profesionales del periodismo se encuentran: los medios de comunicación masiva (impresos, radiales, televisivos y digitales), entornos empresariales, sociales y políticos; publicaciones periódicas generales y especializadas de carácter nacional, provincial y municipal; emisoras de radio y televisoras nacionales, provinciales, municipales e internacionales; agencias de noticias nacionales e internaciona- les; instituciones culturales y de otros sectores nacionales e internacionales, entidades de la red editorial; sitios web y otros entornos de producción digital; entidades docentes, científicas, proyectos institucionales, sociales y escenarios emergentes mediáticos y extramediáticos.

Una de las funciones del profesional del periodismo es desempeñar diferentes roles en escenarios de producción infocomunicativa, tradicionales e hipermediales, para lo cual se articulan desde el primer año de la carrera un grupo de disciplinas.

\section{3- LA DISCIPLINA COMUNICACIÓN HIPER- MEDIA, TECNOLOGÍA Y SOCIEDAD PARA LA FORMACIÓN DE LOS ESTUDIANTES DE PE- RIODISMO}

La sociedad de la información barrió con los patrones tradicionales de generación de contenidos para la prensa [...] los flujos de comunicación, antaño unidireccionales y demasiado predecibles, emergen ahora desde todas partes e irrumpen prácticamente sin jerarquías dentro del discurso público [...] Es una transformación impensable al margen del impacto de las nuevas tecnologías, que demanda ajustar estratégicamente el ejercicio de la economía, la cultura y la política a las lógicas de una sociedad red (Garcés, citado en Molina, 2018: 127).

El periodismo, particularmente, ha tenido que atenerse a las condiciones de la convergencia digital que supone procesos de descentralización y emergencia de nuevos actores, escenarios y procesos de usos de la comunicación y la información, así como la remediación constante de dispositivos, discursos y responsabilidades de las actividades asociadas a la condición del medio de comunicación periodísticos.

En particular, el escenario cubano, avanza hacia una progresiva y compleja densificación de las prácticas, infraestructuras, dimensiones interpersonales, institucionales, gubernamentales, políticas, locales y trasnacionales de la comunicación digital en red; a la par de los retos propios de la consecución de un modelo de desarrollo social propio, equitativo, sostenible e independiente que marcan el desenvolvimiento del sistema comunicativo cubano a lo largo de su historia.

Desde los Centros de Educación Superior la disciplina Comunicación Hipermedia, Tecnología y Sociedad se articula a partir de un enfoque formativo que equilibra teoría y práctica para evitar visiones instrumentales de los saberes o abstracciones absolutas respecto a las prácticas habituales. Hoy, en correspondencia con las demandas antes identificadas, se propone un diseño de la disciplina transversal a la carrera, entendiendo a las TIC digitales en red como media- 
ciones esenciales a todo proceso de producción, circulación y consumo de información, pero con particular atención a las necesidades propias del área de conocimiento.

La disciplina Comunicación Hipermedia, Tecnología y Sociedad contiene 3 asignaturas en su currículo base. Estas asignaturas son: Introducción a Comunicación Hipermedia I, Comunicación, Tecnología y Sociedad y Periodismo Hipermedia. También posee dos asignaturas en el currículo propio: Introducción a Comunicación Hipermedia II y Taller de Producción Hipermedia.

Las asignaturas de la disciplina Comunicación Hipermedia, Tecnología y Sociedad pone énfasis en la realización de múltiples ejercicios vinculados a la elaboración de productos periodísticos que respondan a las características de la comunicación en red, uno de los objetivos que deben vencer los educandos al finalizar las asignaturas y que combinen a su vez con la capacidad de comprensión crítica de su entorno a través de la mediación de las TIC.

Esta disciplina resuelve como problemática fundamental las transformaciones tecnocomunicativas de los entornos digitales sociales, los usos sociales y las competencias infocomunicacionales de los usuarios y los escenarios locales, grupales, institucionales y trasnacionales de su desarrollo. En particular, las redimensiones de los discursos, las prácticas, las instituciones, los marcos regulatorios y los procesos de convergencia mediática en general de la producción periodística.

En este sentido se propone cubrir un espectro que va desde los conceptos y competencias profesionales necesarias para el periodista hipermedia, el tratamiento y recuperación de recursos de información, la aprehensión de las particularidades narrativas del lenguaje, la visión holística de los procesos editoriales para los medios en ambientes digitales, así como comprensión los impactos de las Tecnologías de la Información y la Comunicación en todos los ámbitos sociales.

Entre los objetivos fundamentales de la disciplina Comunicación Hipermedia, Tecnología y Sociedad, el estudiante debe ser capaz de: elaborar productos periodísticos atendiendo a las posibilidades expresivas de Internet, con una comprensión sobre el papel de la comunicación hipermedia ante situaciones comunicativas diversas con un enfoque integral, inclusivo y de gestión del desarrollo como objetivo.

Una de las asignaturas que comprende la disciplina motivo de este estudio es Periodismo Hipermedia. Esta asignatura se imparte en el primer semestre del segundo año de la carrera, por eso, al finalizar el segundo año el estudiante debe ser capaz de: elaborar mensajes periodísticos audiovisuales desde una perspectiva inter y transdisciplinar, logrando la apropiación del lenguaje y las técnicas expresivas en entor- nos tradicionales e hipermediales, desde una postura ética, con enfoques inclusivos y de identidad nacional. También los alumnos deben realizar cobertura para los entornos tradicionales e hipermediales ante situaciones comunicativas diversas generadas desde los propios medios y el contexto social, político, económico y cultural, utilizando los conocimientos adquiridos.

\section{4- ESTRATEGIA PARA LA FORMACIÓN DE LOS ESTUDIANTES DE PERIODISMO ANTE EL DESARROLLO DE LAS TIC.}

\subsection{1- LAS TICS Y EL PERIODISMO HIPER- MEDIA EN FUNCIÓN DE LOS ESTUDIANTES DE LA CARRERA}

El Periodismo Hipermedia es una modalidad profesional del periodismo porque modifica los tres procesos básicos en los que se basa esta profesión: la investigación, la producción y la difusión. La producción y difusión de noticias se reforman a partir de herramientas digitales y de la emergencia de nuevos medios que emplean las plataformas virtuales para publicar sus contenidos.

El Periodismo Hipermedia es aquel que se ejerce desde y para la producción de contenidos en diversos formatos hipermedia. Se caracteriza por un lenguaje periodístico que aún está construyendo su propia gramática, lo que supone nuevas competencias profesionales para enfrentar este proceso y/o producto comunicativo. No hace referencia solo a los periodistas tradicionales que producen textos para publicarlos en la red, sino en mayor grado, se refiere a aquellos que tienen competencias en el manejo de herramientas tecnológicas y las integran a su ejercicio profesional para producir contenidos con formato hipermedial (Alayón, 2017).

La asignatura Periodismo Hipermedia que se imparte en la Universidad de Camagüey Ignacio Agramonte y Loynaz a los estudiantes de segundo año de la carrera proporciona las bases teóricas de los principales cambios introducidos en los lenguajes y rutinas productivas. También le permite a los estudiantes relacionarse de forma práctica con la producción del periodismo "para y con Internet", y desarrollar la concepción del trabajo multidisciplinar como la forma de afrontar los retos introducidos por las TIC.

Hoy en día la práctica del periodismo hipermedia, dirigida a un público cada vez más exigente, con mayores facilidades para crear e interactuar, ha alterado esa cultura profesional que identificó durante tanto tiempo a los informadores por excelencia. No obstante en el ciberperiodismo adquieren gran importancia los valores y principios éticos. La veracidad constituye un componente fundamental con el que debe contar toda información, para brindar a las audiencias 


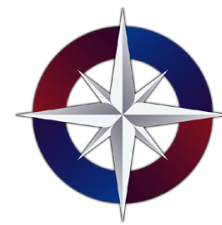

$\begin{array}{lllllll}R & E & V & I & S & T & A\end{array}$ aquellos elementos que le permitan tomar decisiones, forjar sus propios criterios. Por otro lado el hecho de que parte de las fuentes de información se encuentren en la red, sin posibilidad de verificación en algunos casos, demanda la puesta en práctica de confirmaciones y procesos de comprobación a veces más complejos que con las fuentes tradicionales.

"En la nueva sociedad de redes, cada ciudadano es un "periodista" en potencia. Delante de su pantalla de ordenador, de teléfono, o de su tableta táctil, el internauta que domina los recursos de la Web no es considerado inferior al periodista profesional. Es más, le disputa su estatus de supremacía. Por su parte, los medios dominantes no dejan de alentar a los internautas que se conviertan en "periodistas«. Constantemente le piden que suban a sus páginas web fotos, videos o comentarios sobre cualquier asunto del que hayan sido testigos: un acontecimiento político o meteorológico, un accidente, una manifestación callejera, etc. De ahíla gran interrogación que surge en relación con la identidad del periodismo y la validez de la información" (Ramonet, 2011).

Por eso, ante las nuevas y crecientes posibilidades comunicativas, los medios de comunicación en Cuba y la universidad, deben trabajar en función de que los estudiantes se familiaricen, y practiquen con la creación los contenidos hipermediales que respondan a las cambiantes exigencias del entorno digital, como único camino para garantizar la visibilidad y perdurabilidad de los mensajes en espacios internacionales.

Actualmente el debate académico y profesional en torno a las cualidades que debe poseer el denominado periodista digital se enriquece por la diversidad de criterios. Según el periodista y teórico español Ramón Salaverría "el periodista digital debe ser ante todo periodista, que tenga como norte la información y el servicio al público, apoyándose en la técnica y el soporte de difusión para lograr sus objetivos" (Salaverría, 2008).

El periodismo hipermedia rompe con la individualidad de antaño, cuando las empresas se limitaban a un solo soporte. Ahora, estas empresas que tienden a la concentración empresarial, buscan multiplicar su presencia en todos los medios a través del múltiple aprovechamiento de los recursos materiales y humanos que trae la convergencia, así como la unificación de redacciones. La redacción integrada es un fenómeno particular dentro del macrofenómeno de la convergencia.

La tecnología digital favorece la integración de funciones que antes estaban separadas en el proceso de producción de informativos, por lo que los periodistas asumen las tareas de grabación y edición (hasta hace unos años separadas), y se incrementa la automatización de tareas y servicios. El tra- bajo se divide en compartimentos cada vez más reducidos, de manera que algunos profesionales llegan a cuestionarse su propia función de reporteros. Hay menos redactores que buscan y generan noticias, y cada vez son más quienes se dedican a elaborar lo que se recibe de agencia o a través de otras fuentes.

\subsection{2- RECOGIDA DE LA INFORMACIÓN EN LOS MEDIOS ONLINE PARA EL PERIO- DISMO HIPERMEDIA}

En el escenario digital actual ha cambiado el papel protagónico de los medios de presentar al público los temas de la realidad sobre los cuales deben formarse diversas opiniones, ahora las audiencias han ganado voz del otro lado del ordenador y cuando la noticia se publica en sitios de redes sociales como Facebook o Twitter, muchas de las organizaciones informativas se nutren de los temas relevantes que circulan en estas plataformas para conformar sus agendas.

Sin embargo, los sitios de redes sociales más allá de ser mucho más dinámicos y de dar a conocer la diversidad de opiniones de la sociedad con respecto a cualquier tema, estos espacios no remplazan los principios básicos de la investigación periodística de los cibermedios. Prácticas tradicionales como la determinación de los valores noticia, el empleo de diferentes fuentes, incluyendo la confianza en las institucionales, la confirmación de la información, permiten que la agenda de los medios gane legitimidad.

Los valores noticia constituyen el componente imprescindible de la noticiabilidad. Entre los valores noticia más relevantes se encuentran: novedad; originalidad e imprevisibilidad ; evolución futura de los acontecimientos; importancia y gravedad; proximidad geográfica del hecho a la sociedad; magnitud por la cantidad de personas o lugares implicados; jerarquía de los personajes e inclusión de desplazamientos. Según las cualidades que el acontecimiento presenta en relación con los procesos destacan: comprensión e inteligibilidad; credibilidad; brevedad, periodicidad; exclusividad y la noticia como resultado de una ideología de la información.

De esta manera se describe al periodista que produce para medios digitales, como un profesional capaz de salir a la calle o entrar a la red para buscar la noticia de último momento y transmitirla al usuario, convirtiéndose a su vez, ya sea en la sala de redacción o en el mismo lugar de los sucesos acompañado de la tecnología, en un cartógrafo que dibuja el camino a recorrer por el navegante mediante un uso racional e intencionado de los recursos del periodismo hipermedia. 


\subsection{3- EL PERIODISMO HIPERMEDIA, UN NUEVO MODELO DE PRODUCCIÓN PARA LOS MEDIOS ONLINE}

Desde la implementación del Plan E en la Carrera de Periodismo en la Universidad de Camagüey Ignacio Agramonte y Loynaz, a partir del año 2017, la diversidad de enfoques contenidos en los programas ha dependido en gran medida del contexto social en el que han sido producidos. La mayor confluencia de capacitación periodística sobre las nuevas tecnologías se centra en la disciplina Comunicación Hipermedia, Tecnología y Sociedad. Esta disciplina, integra los saberes en torno a los nuevos escenarios infocomunicativos y constituye el eje articulador de todos los lenguajes de la profesión.

El modelo ideal de periodismo digital es un referente importante basado en: profundidad, voces múltiples, integración multimedia y participación de los usuarios. Estos constituyen valores esenciales en la mayoría de los casos. Entre los recursos de los que se vale el Periodismo Hipermedia se encuentran: la hipertextualidad, la multimedialidad y la interactividad.

El éxito de la segunda generación de Internet puede resumirse en tres factores: un acceso a centenares de aplicaciones multimedia gratuitas, el poder de la inteligencia colectiva, la constante participación de los usuarios. En la era de la participación, los consumidores ya no quieren limitarse a recibir información sobre un nuevo producto o servicio, sino que, además, el público consumidor quiere interactuar en los nuevos medios de comunicación pasando a formar parte del proceso informativo.

Dichos recursos fueron innovaciones técnicas y conceptuales que Internet reunía y sugerían un nuevo modelo de periodismo basado en: el fin de los límites temporales y espaciales de los productos informativos, el desvanecimiento de los formatos mediáticos y el nacimiento de la narración multimedia, una combinación de texto, fotografías, infografías animadas, piezas de audio y de video y una implicación activa de la audiencia en el consumo y producción de noticias, a través de la personalización del producto, los foros de opinión, los e-mails de contacto de los periodistas y nuevos espacios abiertos para la publicación en los sitios web de los medios como los blogs.

1.4.4- LOS BLOGS DIGITALES COMO ESTRATEGIAS PARA LA FORMACIÓN DE LOS ESTUDIANTES DE PERIODISMO EN ANTE EL DESARROLLO DE LAS TIC.

Un blog (también bitácora digital, web blog, o weblog) es un sitio web en el que uno o varios autores publican cronológicamente textos o artículos, apareciendo primero el más reciente, y donde el autor conserva siempre la libertad de dejar publicado lo que crea pertinente. También suele ser habitual que los propios lectores participen de forma activa a través de los comentarios. Un blog puede servir para publicar ideas propias y opiniones de terceros sobre diversos temas.

Entre las características técnicas de estos espacios se encuentran: los comentarios. Mediante un formulario se permite, a otros usuarios de la web, añadir comentarios a cada entrada, pudiéndose generar un debate alrededor de sus contenidos, además de cualquier otro intercambio de información. Habitualmente, en cada artículo de un blog, los lectores pueden escribir sus comentarios y el autor darles respuesta, de forma que es posible establecer un diálogo.

También como otro recurso están los hipervínculos o enlaces. Una particularidad que diferencia a los blogs de los sitios de noticias, es que las anotaciones suelen incluir múltiples enlaces a otras páginas web, como referencias o para ampliar la información agregada. Además, y entre otras posibilidades, permite la presencia y uso de: un enlace permanente (permalink) en cada anotación, para que cualquiera pueda citarla, un archivo de las anotaciones anteriores y una lista de enlaces a otros blogs seleccionados por los autores.

El uso o tema de cada blog es particular, los hay de tipo: periodístico, empresarial o corporativo, tecnológico, educativo, políticos, personales, entre otros. Los blogs pueden ser construidos y almacenados usando servicios de alojamiento de blogs dedicados, o pueden ser concretados y accedidos mediante software genérico para blogs, como por ejemplo usando los productos Blogger o Wordpress.

Una de las opciones que permiten los blogs y que más gustan a los usuarios es la posibilidad de inserción de fotografías y vídeos. Estos sitios web son realmente ideales para integrar contenidos periodísticos. Por eso, en la Universidad de Camagüey, a los estudiantes de Periodismo a través de la asignatura Periodismo Hipermedia que se imparte en el segundo año de la carrera se les orientó como Trabajo Final que debían crear blogs periodísticos, desde los cuales subirían trabajos propios, utilizando elementos y recursos hipermediales.

Los blogs se concibieron utilizando el trabajo en grupo pues "el trabajo grupal, permite ejecutar las acciones a partir de la orientación formada. Las funciones fundamentales del maestro, son las de interpretar y coordinar la dinámica grupal y evaluar el aprendizaje" (Colectivo de Autores, 2002: 128). Dichos trabajos periodísticos debían ser de prensa plana, de agencias de noticias cubanas, radiales y televisi- 


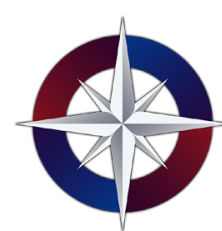

$\begin{array}{lllllll}\mathrm{R} & \mathrm{E} & \mathrm{V} & \mathrm{I} & \mathrm{S} & \mathrm{T} & \mathrm{A}\end{array}$ vas, para coadyuvar a la integración con otras asignaturas del año académico.

La plataforma en que los estudiantes crearon los blogs fue Reflejos, con dominio cubava.cu. Dicha plataforma es de acceso libre, alojada en el dominio.cu y es atendida por los Joven Club de Computación y Electrónica. Reflejos está sustentada en sobre el sistema de gestión de contenidos Wordpress. En Reflejos, los usuarios tienen un espacio accesible donde compartir sus opiniones e intereses. Aunque se puede acceder desde cualquier lugar del planeta, solo pueden registrase usuarios desde Cuba.

Acorde con los objetivos que el estudiante debe vencer en segundo año (Ver Anexo 1), los blogs debían tener los siguientes requisitos:

- $\quad$ Adecuada utilización de audios, videos y abundancia de enlaces hipermediales.

- Atractivo diseño visual, que incluye columnado acorde con el estilo del blog, tipografía y puntaje uniforme.

- Empleo de géneros periodísticos variados e inclusión de géneros periodísticos, propios del Periodismo Hipermedia: dossier multimedia, artículo hipermedial, infografía, el podcsat a través de la plataforma IVOOX y los videoblogs a través de la Plataforma Youtube u otras.

- Utilización correcta de las etiquetas, división por categorías y las fuentes de información.

- Manejo apropiado del lenguaje literario, empleo de recursos lingüísticos y también de los colores.

- Utilización oportuna de una galería de imágenes interactiva.

- El Blog debía tiene interacción con los Sitios de Redes Sociales (Facebook, Twitter, Instagram, plataformas de audio y Linkedin)

- El Blog debía estar distribuido por categorías.

- Debían utilizar mapas interactivos, utilizando la plataforma digital de Sistema de Información Geográfica (SIG) Maphub. Esta plataforma se encuentra alojada en el sitio Maphub.net

- Este sitio debía poseer un adecuado empleo de las etiquetas y las fuentes de información.

- Las fotos que utilizaran debían tener los créditos y los metadatos de la imagen.

De esta manera se crearon cuatro blogs periodísticos (Ver Anexo 2) en función de aplicar los conocimientos. Estas bi- tácoras han quedado como repositorios digitales para que los estudiantes se mantengan publicando y los actualicen con trabajos que otras asignaturas del año académico le orientan.

\section{5- PROPUESTA METODOLÓGICA PARA LA ENSEÑANZA DEL PERIODISMO HIPERME- DIA, UTILIZANDO LOS BLOGS COMO PARTE DE LAS TICS}

Una de las principales contradicciones a la hora de utilizar las Tecnologías de la Información y las Comunicaciones (TICs) en la enseñanza del Periodismo Hipermedia es si es más conveniente enseñar teóricamente las herramientas para utilizar los recursos hipermediales, o si se debe enseñar al estudiante paso a paso a utilizar estas tecnologías desde la práctica y que aprehendan de ellas para su futura vida profesional. Se recomienda desde el punto de vista didáctico - metodológico seguir un sistema de trabajo que, con el propósito de integrar las tecnologías de la información y las comunicaciones a la enseñanza del Periodismo, sea factible en su implementación para los docentes y exitoso en la apropiación de conocimientos y habilidades para los estudiantes.

Esto se explica mejor con la adopción de un plan de superación para el docente y que garantiza la preparación metodológica respecto a las TICs desde la planificación del sistema de clases, dirigido a docentes que pueden no ser usuarios avanzados en el dominio de las TICs, pero sí poseer conocimientos básicos al respecto. Sin embargo, es conveniente destacar que independientemente de que se disponga de avanzadas tecnologías, el profesor debe tener una actitud de director del aprendizaje e ir planteando diferentes cuestiones a resolver por el alumno en un aprendizaje guiado y basado en la resolución autónoma o grupal de los problemas propuestos.

En correspondencia con lo anterior, se demanda que el profesor que imparte Periodismo Hipermedia posea cierta competencia respecto al dominio de la computación, la capacidad de gestión de información en Internet y habilidades básicas de las TICs y el Periodismo. Una vez que el profesor esté capacitado, se habrán creado condiciones para poder implementar de forma efectiva este tipo de tecnología y desarrollar las potencialidades que le puede ofrecer en función del aprendizaje. Para ello se recomienda tener presente la siguiente propuesta:

1. Autopreparación del profesor: Permitiría adquirir el dominio de las bases teóricas y las habilidades prácticas respecto al uso e implementación de los TICs en la clase de Periodismo Hipermedia.

2. Realizar la preparación metodológica de la asigna- 


\section{익 P U B L I C A N D O \\ I S S N $13990-9304$}

tura que imparte: Le permitirá al profesor identificar las potencialidades de las TICs al planificar el sistema de actividades, trabajos extracurriculares, investigativos o simplemente emplearlos en su clase como fuente de análisis de información. Se garantizarán todas las condiciones materiales y cognitivas.

3. Impartición del contenido con ayuda de las TICs: Se deberá mostrar no solo la información obtenida por el profesor, sino mostrar al estudiante cómo gestionarla y utilizarla.

4. Orientación de las actividades a desarrollar por los estudiantes: Debe quedar preciso el objetivo de la actividad (Ver Anexo 3), los materiales a utilizar, dónde encontrar la información y forma de entrega de los trabajos.

5. Valoración del grado de cumplimiento del objetivo: Es la forma de comprobar si se logró la integración de los contenidos, qué resultó positivo y qué fue negativo. Se recomienda además escuchar el criterio particular de los estudiantes sobre la ejecución de las actividades. En este caso, el resultado final fue la publicación de los blogs periodísticos (Ver Anexo 4).

En el paso 1 de la propuesta metodológica se evidencia en el grado de preparación del docente respecto al uso de las TICs luego de haber cursado el Curso de Posgrado sobre Periodismo Hipermedia en el Instituto Internacional de Periodismo José Martí, apoyado en la autogestión de la actividad cognitiva. Dentro de los temas principales de este curso destacan el marco teórico y conceptual del Periodismo Hipermedia internacionalmente y en Cuba, Estrategia de Sitios de Redes Sociales, Periodismo Móvil y herramientas analíticas de posicionamiento web.

El paso 2 de la propuesta metodológica se manifiesta en la planificación del sistema de clases de la asignatura que imparte el docente teniendo en cuenta el momento propicio para la integración entre las TICs y el contenido que impartirá. El paso 3 de la propuesta metodológica se materializa en la impartición de la clase donde se orienta a los estudiantes sobre las particularidades de las TICs a emplear. Es en este momento es donde ocurre la mayor parte de la actividad para el alumno. Por otra parte se demuestra el cumplimiento del paso 4 de la propuesta, pues los estudiantes son capaces de vencer los objetivos del trabajo que se les orientó.

En cada uno de los casos, al aplicar el paso 5 de la propuesta metodológica se evaluó el desempeño de los estudiantes, se realizaron reflexiones con ellos sobre sus opiniones en la aplicación de este tipo de ejercicio y la valoración por parte del profesor respecto al cumplimiento del objetivo de la actividad, así como la identificación de los elementos positivos y negativos. La propuesta presentada se encuentra en la fase de implementación, pero ya se pueden expresar resultados cualitativos en función del aprendizaje de los estudiantes de la carrera de Periodismo en la Universidad de Camagüey, al poder utilizar la diversidad de fuentes de información y poder constatar que los blogs están activos y con abundancia de recursos propios del Periodismo Hipermedia.

Paralelamente a lo relacionado con lo curricular, y en función de potenciar mucho más el uso de las TICs en la enseñanza del Periodismo, se han dado pasos en el Departamento de Periodismo y Comunicación Social que refuerzan esta implementación que incluyen la superación de los docentes, por ejemplo: la Estrategia Tecnológica del Departamento incluye tanto la superación del personal docente como la formación de habilidades específicas en los estudiantes de la especialidad, así como los mecanismos para su control y evaluación.

\section{CONCLUSIONES}

Desde la implementación del Plan E en la Carrera de Periodismo de la Universidad de Camagüey Ignacio Agramonte y Loynaz, a partir del año 2017, la diversidad de enfoques contenidos en los programas ha dependido en gran medida del contexto social mediado por prácticas infocomunicativas, a tenor con el desarrollo de las TICs en la que ha estado inmerso el país para informatizar la sociedad, desde hace ya algunos años. Estos programas académicos son el resultado de la conjugación de prácticas y teorías históricamente asentadas con nuevos saberes que responden a la evolución de la llamada sociedad de la información y el conocimiento.

La mayor confluencia de capacitación periodística sobre las tecnologías de la información y la comunicación se centra en la disciplina Comunicación Hipermedia, Tecnología y Sociedad. Esta disciplina, integra los saberes en torno a los nuevos escenarios infocomunicativos y constituye el eje articulador de todos los lenguajes de la profesión, así como entre los diálogos inter y transdisciplinares. Los estudiantes reciben las herramientas tecnológicas necesarias a lo largo de la carrera, que potencien los conocimientos y habilidades en el uso de las TIC para la praxis infocomunicativa en aras de la innovación y el desarrollo así como para el análisis crítico de las estrategias de su aplicación en Cuba y el mundo.

La asignatura Periodismo Hipermedia que se imparte para segundo año, específicamente en el segundo semestre tiene, desde el punto de vista teórico y práctico vínculos muy estrechos con asignaturas como Periodismo en Radio y Televisión II, Agencias de Noticia y la Optativa III Locución y Conducción de Espacios Informativos Audiovisuales, de ahí la importancia de organizar este ejercicio integrador para estrechar los lazos entre las diferentes asignaturas afi- 
nes.

La importancia de la asignatura Periodismo Hipermedia radica en que a partir de los blog, creados por los equipos, los estudiantes pueden subir los trabajos periodísticos creados por ellos en las distintas asignaturas. Además, los educandos deberán manifestar sus habilidades para compartir estos contenidos periodísticos en distintos sitios de redes sociales como Facebook, Twitter, Instagram, entre otros. Para la formación de los estudiantes de segundo año de la carrera, resulta vital este ejercicio pues, los alumnos se apropian de los lenguajes y prácticas propias del periodismo digital y también de las distintas herramientas digitales que facilitan el trabajo para y con Internet. 


\section{REFERENCIAS BIBLIOGRÁFICAS:}

Addine, F. (2014). Didáctica General. La Habana: Editorial Pueblo y Educación.

Alayón, L. (2017). Las rutinas de producción hipermedia en Vanguardia web. Trabajo de Diploma en opción al Título Licenciado en Periodismo. Universidad Central de Las Villas, Villa Clara, Cuba.

Alonso, M. (2000, enero-junio). La investigación de la comunicación en Cuba: préstamos teóricos para un itinerario singular. No 20-21 .pp. 45

Ávila, R. (2001). Guía para elaborar la tesis. Metodología de la Investigación. Cómo elaborar la tesis y/o investigación. Ejemplos de diseños de tesis y/o investigación. Perú, Lima: Estudio y Ediciones RA.

Bermúdez, R. (Comp.) (2002). La labor educativa en la escuela. La Habana: Editorial Pueblo y Educación.

Bermúdez, R. (Comp.) (2005). El pensamiento de Fidel Castro sobre educación. La Habana: Editorial Pueblo y Educación.

Betto F. (2014). El papel del educador en la formación política de los educandos. Conferencia presentada en Evento Internacional en La Habana.

Diz, E. (2011). Reubicar el futuro. Las mediaciones entre la cultura profesional y las lógicas de producción periodística para la web. Estudio de casos: Granma y Trabajadores. Tesis de doctorado. Universidad de La Habana, La Habana, Cuba.

Edo, C. (2001). El lenguaje periodístico en la red: del texto al hipertexto y del multimedia al hipermedia. Sevilla: Comunicación Social.

Engels, F. (1979). Generalidades. En: Anti-Dühring. Ciudad de La Habana: Editorial Pueblo y Educación.

Fraga, D. (2018). La calidad en la formación integral del profesional. Conferencia en formato digital del MES.

Guadarrrama, P. (2012). Dirección y Asesoría de la Investigación Científica. La Habana: Editorial de Ciencias Sociales.

Hernández, R. (2006). Metodología de la Investigación. México: McGraw-Hill.

Horruitiner, P. (2009). La universidad cubana: el modelo de formación. La Habana: Editorial Universitaria.

Linares, M. y Santovenia, J. (2012). Buenas prácticas: Comunicar e Informar. La Habana: Editorial Academia.

Ministerio de Educación Superior (MES) (2013). Perfeccionamiento del sistema de gestión del proceso de formación integral de los universitarios en el eslabón de base.

Ministerio de Educación Superior (MES) (2017) Documento base para el diseño de los Planes de estudio "E".

Ministerio de Educación Superior (MES) (2018) Reglamento de Organización Docente de la Educación Superior.

Molina L. (2018 enero-abril). La convergencia periodística en la prensa cubana. Nuevos roles profesionales para periodistas y académicos. En: Islas. Universidad Central «Marta Abreu» de Las Villas. Villa Clara

Plasencia, A. (1973). Método y Metódica Históricos. La Habana: Editorial de Ciencias Sociales.

Ramonet, I. (2011). La explosión del periodismo. De los medios de masas a la masa de medios. La Habana: Editorial José Martí

Rodríguez G; Gil, J. y García E. (1996). Metodología de la Investigación Cualitativa. España: Editorial Aljibe.

Sabino, C. (1994). Cómo hacer una tesis. Editorial: Panapo. Caracas.

Salaverría, R. (2008). Periodismo integrado: Convergencia de medios y reorganización de redacciones. España: Sol90.

Sánchez, I. (2012). Sospechas y disidencias. Una mirada cubana en la red. Casa Editora Abril: La Habana.

Suárez, L. (2018). La labor educativa en el colectivo de año. El rol de la estrategia educativa de la comunidad universitaria del año académico para su concreción. Conferencia presentada en el Diplomado para profesores jóvenes. Universidad de Matanzas: Matanzas.

Tamayo, M. (1999). Serie Aprender a investigar. Módulo 2. La Investigación. Editores LTDA: Santa Fe de Bogotá. 


\section{ANEXOS}

ANEXO 1- OBJETIVOS GENERALES DE LA ASIGNATURA PERIODISMO HIPERMEDIA, QUE EN CORRESPONDENCIA CON EL PLAN DE ESTUDIOS E SE IMPARTE EN EL PRIMER SEMESTRE DEL SEGUNDO AÑO

- Analizar la evolución que ha tenido la producción periodística "para y con Internet", desde su surgimiento hasta la actualidad.

- Caracterizar los estándares de producción del Periodismo hipermedia, tanto desde la reflexión teórica como desde la práctica periodística en Internet.

- Desarrollar en los estudiantes las habilidades y saberes necesarios para elaborar productos periodísticos hipermediales, tanto en condiciones normales como en momentos de desastres naturales o situaciones de conflicto.

- Estructurar el trabajo en equipo y argumentar la importancia de los grupos multidisciplinarios para enfrentar los retos que suponen la convergencia de profesiones y tecnologías.

\section{ANEXO 2- CARACTERIZACIÓN DE LOS CUATRO BLOGS DE LOS ESTUDIANTES DE PERIODISMO}

\section{BLOG NÚMERO 1}

Título: Ahora Periodistas

Dirección electrónica: estudiantesuc.cubava.cu

Integrantes: Marianne Portuondo Méndez, Idaylén Rodríguez Rodríguez, Dailén Fenollar Alemán, Legna Caballero Pérez, Alejandro García Gutiérrez, Elena Rosales Caballero.

\section{BLOG NÚMERO 2}

Título: Muchas voces

Dirección electrónica: periodistas.cubava.cu

Integrantes: Lázaro Torres Hernández, Isbely Hernández Barrizonte, Abraham Sierra Quiros, Reinier Hernández González.

\section{BLOG NÚMERO 3}

Título: Miradas al desnudo

Dirección electrónica: universitarios.cubava.cu

Integrantes: Gabriel Peña Ramírez, Yilenis Pérez Sanabia, Dayana Menzoney Justiz , Melissa Lorenzo Comesaña, Lianet Vega Borrero, Enamnuel Santos Rodríguez y Haniel Valdés Velázquez.

\section{BLOG NÚMERO 4}

Título: Multiverso

Dirección electrónica: f34r.cubava.cu

\section{ANEXO 3- CLASE DE ORIENTACIÓN DE LOS BLOGS PERIODÍSTICOS}

El objetivo de esta actividad es: Crear blogs periodísticos, utilizando las tecnologías de la información y las comunicaciones, para que los estudiantes se apropien de los lenguajes y prácticas propias del periodismo digital que facilitan el trabajo para y con Internet.

El grupo debe dividirse en 4 equipos (seis integrantes) y se asignará un blog para cada equipo. El profesor seleccionará el Jefe de cada equipo que será el administrador.

¿Qué evaluará el profesor?

1 Administrador: Es el máximo dirigente, es como el director en un medio de prensa y tiene la potestad para decidir, valorando la opinión de su equipo el título del blog, si se publica o no el trabajo y puede sugerir los temas, el tipo de trabajo, enlaces, fotografías, videos, socialización en redes sociales y otros elementos hipermediales. $\mathrm{Al}$ administrador se le evaluará por el trabajo desempeñado como líder de un proyecto, su capacidad para trabajar en equipo y como responsable de la calidad que tenga el producto final. Los materiales periodísticos a utilizar son los creados por ustedes en otras asignaturas del año académico. Cualquier información teórica que necesiten pueden buscarla en la bibliografía digital orientada en cada una de las clases y apoyarse en la consulta de la página web de la Universidad.

2 estudiantes que trabajen en el diseño formal y visual: Estos estudiantes serán los diseñadores, encargados de adecuar la plantilla que consideren conveniente, determinar los colores, el slogan, el columnado, la tipografía, el tamaño o puntaje de los caracteres, nivel de interacción con los usuarios y cualquier otro elemento visual que requiera el blog.

3 estudiantes que trabajen en el contenido y en los elementos hipermediales: Estos estudiantes funcionarán como periodistas-reporteros. Los géneros periodísticos que 
podrán realizar son: noticias (género información con las características que estudiamos en clase, utilizando la estructura dramática, la pirámide invertida o el Cubo de Rubik.). Pueden utilizar otros géneros típicos del periodismo hipermedia (infografía periodística, podcast, mapas interactivos, videoblogs, reportaje y dossier multimedia)

El día de la exposición los equipos deben mostrar los productos finales y estar preparados para debatir acerca de cómo elaboraron el blog y qué tuvieron en cuenta para hacerlo. El profesor medirá en este ejercicio las habilidades de análisis y razonamientos así como la capacidad de trabajar en equipo. Los estudiantes deben ser creativos. Independientemente que el trabajo sea en equipos, la evaluación será individual en relación con el desempeño de cada estudiante. 